\title{
Multiple Target Detection using Bayesian Learning
}

\author{
Sujit Nair, Konda Reddy Chevva, Houman Owhadi and Jerrold Marsden
}

\begin{abstract}
In this paper, we study multiple target detection using Bayesian learning. The main aim of the paper is to present a computationally efficient way to compute the belief map update exactly and efficiently using results from the theory of symmetric polynomials. In order to illustrate the idea, we consider a simple search scenario with multiple search agents and an unknown but fixed number of stationary targets in a given region that is divided into cells. To estimate the number of targets, a belief map for number of targets is also propagated. The belief map is updated using Bayes' theorem and an optimal reassignment of vehicles based on the values of the current belief map is adopted. Exact computation of the belief map update is combinatorial in nature and often an approximation is needed. We show that the Bayesian update can be exactly computed in an efficient manner using Newton's identities and the detection history in each cell.
\end{abstract}

\section{INTRODUCTION}

Multiple target search and tracking [1], [2], [3], [4] are important elements of a surveillance system. In this setting, one is interested in determining the number as well as the state of targets. Radar and sonar based tracking of objects for air traffic control and navigation are some of the applications of multiple target search and tracking. Search and tracking are key elements for marine search and rescue (SAR) missions using autonomous vehicles [5]. In order to successfully achieve these goals, one needs to effectively extract useful information about the target's state from observations. When targets are not detected, they should be searched for and when a target is found, it should be tracked.

In this paper, we restrict out attention to search (or detection) of an unknown number of stationary targets using measurements from multiple search vehicles. In search theory, one in interested in finding the best way to search for a single object or multiple objects when the possible locations of these objects are drawn from a probability distribution and the search resources like time and fuel are limited. The first studies in search theory are attributed to B. O. Koopman and his group at the Antisubmarine Warfare Operations Research Group (ASWORG) during the second world war [6]. Koopman and his colleagues mainly treated the search problem as an area coverage problem. This work was later generalized by L. D. Stone [7], [8], [9] where the notion of probability of detection was introduced and optimal resource allocation was studied.

Sujit Nair, Houman Owhadi and Jerry Marsden are with Control and Dynamical Systems, Caltech, Pasadena, CA 91125, USA \{nair, owhadi, marsden\}@cds. caltech.edu

Konda Reddy is with Control Systems, United Technologies Research Center, E. Hartford, CT, 06108, USA ChevvaKReutrc.utc. com
A typical search problem is tackled by discretizing the search area into a number of cells (need not be uniform) where each cell is associated with a prior probability of containing a target. The target information can, thus, be described using a probability or belief map [10], [11], [12], [13], [14], [15] whose entries are the probabilities for finding targets in the corresponding cell. In other words, the belief map is a gridded representation of the environment. The search agents are initially assigned to the cells based on the prior information about the targets. As they move through the search area and make observations, the belief map is updated using Bayesian estimation. Based on the values of the belief map, the search algorithm determines the cells to which the search agents should be reassigned. In this paper, we consider that the cells are not independent, i.e., if a measurement is made in a particular cell $i$, it affects the belief map values for all the remaining cells. We assume that there are more than one target and associate a probability of detection $p_{d}$ and probability of false alarm $p_{f}$ with the search vehicle sensors. Note that in our setting, $p_{d}$ and $p_{f}$ are for a single measurement instant. Our approach differs from some related work in the following manner. Bertucelli and How [16] assume that the cells are independent and each cell has uncertain probability which may arise due to sensor ambiguity. The authors consider multiple targets and present a method to embed this uncertainty in the search algorithm. In another related work, Bourgault and coworkers [10] consider a single target and assume $p_{f}=0$. However, the cells are not independent and a Markov model for target motion is assumed.

Multiple target detection and tracking can be regarded as generalization of the recursive Bayesian filter. Computing this nonlinear filter is challenging due to its combinatorial nature and usually it is approximated. An important research theme in multiple target detection is the development of efficient computational techniques to approximate the exact solution of the Bayesian state estimate (see [17] and the references therein). In most realistic situations, the number of targets is not normally known and hence needs to be efficiently estimated [18]. In this paper, we consider the problem of detecting an unknown number of stationary targets. In that case, we compute the belief map for the region and for the estimated number of targets. This is in contrast with [17] where the intensity function (density of the mean count) of the set of targets is propagated. This idea was further generalized in [19] to propagate the distribution of the number of targets and the intensity function. The use 
of intensity function propagation to estimate the number of targets as well as the target states was numerically illustrated in [20] and [21]. Though the detection problem is simpler in nature than the tracking problem, we would like to emphasize that the exact computation of the Bayesian update becomes increasing challenging as the number of unknown targets and the grid size increases. The main motivation for this work is to develop fast, computationally efficient techniques for exact computation of the Bayesian update.

The main contributions of the paper are as follows. We have demonstrated optimal Bayesian updates for detecting unknown number of targets in a given search region. The belief map for the region as well for the estimate of number of targets is propagated. The belief states of the system grows exponentially and usually cannot be solved exactly. However, we show that we can indeed solve the problem in a computationally efficient way by using the detection history in each cell. We also show that using Newton's identities from the theory of symmetric polynomials [22] helps us to exactly update the belief map. A similar application of Newton's identities [23] was brought to our notice recently. In this paper, the authors use Newton's identities (called as Newton-Girard identities in the paper) to reduce the computational complexity to update the belief map for the number of targets.

The paper is organized as follows. In $\S I I$, we formulate the search problem given a fixed number of search vehicles and targets. In $\S I I I$, we propose a new way to calculate the belief map in a computationally efficient manner. We also present a novel application of Newton's identities from the theory of symmetric polynomials to exactly calculate the belief map. Simulation results are presented in $\S I V$.

\section{Problem Formulation}

The main problem we are interested in is the following: Given a search area with a fixed number of stationary targets and search vehicles, design computationally efficient strategies for detecting all the targets. More precisely, consider a search area that is divided into $n$ cells as shown schematically in Fig. 1. Note that we do not require any specific geometry for the cells in the region. All we need is division of the region into finite number of cells with nonzero areas. Let $V$ be the total number of search vehicles. We will consider two cases. In one case, the number of targets is known apriori. In the second case, the number of targets is not known. However, we assume that there is an upper bound $\bar{T}$ for the number of targets in the region. We assume that the targets are stationary. We adopt a simple model for the vehicle dynamics. At each time step, the vehicles can jump to any other cell or remain in their current cell. Each cell can only be occupied by a single search vehicle and/or a single target. The vehicles detect a target with probability $p_{d}$. We will also consider false alarms with probability $p_{f}$. At each time step, the vehicles send their detection data, i.e., detect or no detect, to a central manager, that updates the

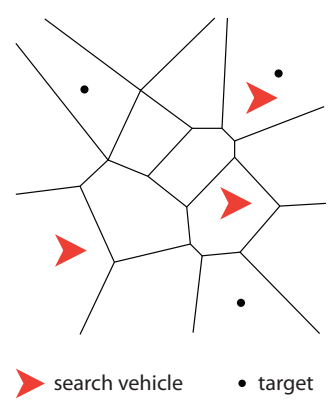

Fig. 1. A schematic of the search grid with fixed number of search vehicles and targets

belief map based on the vehicle measurements. Note that we do not make any assumptions as to when the central manager receives these observations. It need not be the case that observations are made at regular intervals and communication delays are incorporated in our setting. The belief map is a vector of numbers, $P\left(T_{j}\right), j=1, \ldots, n$, where $P\left(T_{j}\right)$ denotes the probability that the target is in cell $j$. The vehicles are then reassigned to new cells based on the updated belief map. There are several ways to reassign the vehicles to new cells. In this paper, we adopt an ideal policy where the vehicles are reassigned or teleported to cells that correspond to the maximum values of the belief map. When we have $m$ vehicles, we assign them to cells with $m$ largest values for the belief map. Though this reassignment scheme is not entirely realistic, it provides lower bounds for the detection times. In order to make sure that vehicles are not infinitely stuck in a cell, we do the following. We set an upper threshold $P_{u}$ and a lower threshold $P_{l}$. If the belief value in a particular cell $j$ is such that $P\left(T_{j}\right)>P_{u}$ or $P\left(T_{j}\right)<P_{l}$, then the cell $j$ is removed from our list of cells, i.e., vehicles are no longer assigned to this cell.

In order to illustrate the application of Bayes' theorem for the search problem, consider the simple case of a single target and a single search vehicle in a grid of size $n$. The essential idea is to update the belief map at each time step based on the measurements made by the vehicles. We assume that the initial target distribution is uniform, that is, $P\left(T_{j}\right)^{0}=$ $1 / n$ for all $j$. Suppose that the vehicle is in cell $i$ and that $d_{i}$ is the detection data. That is, $d_{i}=1$ if the vehicle detects and $d_{i}=0$ if the vehicle fails to detect. Then, given the detection data $d_{i}$, Bayes' theorem can be used to update the belief map:

$$
P\left(T_{j}\right)^{1}=P\left(T_{j} \mid d_{i}\right)=\frac{P\left(d_{i} \mid T_{j}\right) P\left(T_{j}\right)^{0}}{P\left(d_{i}\right)}
$$

where

$$
P\left(d_{i}\right)=\sum_{k=1}^{n} P\left(d_{i} \mid T_{k}\right) P\left(T_{k}\right)^{0}
$$

Note that

$$
\begin{aligned}
P\left(d_{i} \mid T_{j}\right)= & \left(p_{d} \delta_{i j}+p_{f}\left(1-\delta_{i j}\right)\right) d_{i} \\
& +\left(\left(1-p_{d}\right) \delta_{i j}+\left(1-p_{f}\right)\left(1-\delta_{i j}\right)\right)\left(1-d_{i}\right) .
\end{aligned}
$$


Equation (1) can be easily extended to the case of multiple vehicles. Before we do that, we introduce some notation. Let $P\left(T_{i_{1}, \ldots, i_{j}}\right)$ denotes the probability of the event $T_{i_{1}} \cap \ldots \cap T_{i_{j}}$. Here it is assumed that the indices $i_{1}, \ldots, i_{j}$ are distinct.

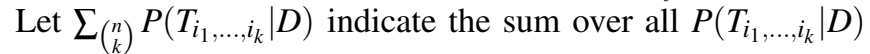
for $\left(\begin{array}{l}n \\ k\end{array}\right)$ ways of choosing indices $i_{1}, \ldots, i_{k}$ from the set $\{1, \ldots, n\}$, where $D$ is some detection data.

One observes that if there are $k$ targets, then the belief map consists of $\left(\begin{array}{l}n \\ k\end{array}\right)$ numbers given by $P\left(T_{i_{1}, \ldots, i_{k}}\right)$. The number of computations required to update the belief map blows up exponentially as the number of cells and targets increases. For example, for a $50 \times 50$ grid with 5 targets, the belief map is already of the order of $10^{14}$. This is a very important consideration if a particular search strategy needs to be implemented in real time. In the next section, we describe a simple, computationally efficient and exact method to update the belief map. This method is particularly attractive when the number of targets and grid size is large.

\section{Computationally EfFicient Belief Map Update}

The purpose of this section is twofold. First is to give an alternative formulation so as to achieve memory storage savings. Second is to show how to use the theory of symmetric polynomials to reduce the computation significantly. From $\S I I$, we know that the size of the event space for a grid consisting of $n$ cells and $U=k$ targets is $\left(\begin{array}{l}n \\ k\end{array}\right)$ corresponding to

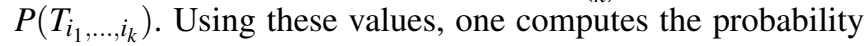
of finding a target in a particular cell $j$ as

$$
P\left(T_{j} \mid U=k\right)=\sum_{\left(\begin{array}{c}
n \\
k-1
\end{array}\right)} P\left(T_{j, i_{1}, \ldots, i_{k-1}} \mid U=k\right) .
$$

We now propose an alternative formulation where one can compute $P\left(T_{j} \mid U=k\right)$ without storing the $\left(\begin{array}{l}n \\ k\end{array}\right)$ numbers $P\left(T_{i_{1}, \ldots, i_{k}}\right)$. We do this by considering the history of detections/no detections in each cell. At each instant of time, vehicles make measurements about targets in their respective cells and report back to a central manager whether they have detected or not detected a target in their respective cells. For each cell, the total number of detections and total number of no detections is stored. Using this information, at each time step, the belief map is constructed as follows: If $d_{i}$ and $m_{i}$ are the total number of detections and no detections respectively in cell $i$ up to that time, the detection data can be written as the following $2 \times n$ matrix

$$
D=\left[\begin{array}{ccc}
d_{1} & \ldots & d_{n} \\
m_{1} & \ldots & m_{n}
\end{array}\right] .
$$

By $D \backslash D_{i}$, we mean the detection data consisting of all cells except the $i^{\text {th }}$ cell.

\section{A. Known Number of Target Case}

We now consider the case when the number of targets is known apriori and is assumed to be $k \leq n$. For the detection data $D$, we can construct the belief map as follows:

$$
\begin{aligned}
& P\left(T_{i} \mid D, U=k\right)=\frac{P\left(D \mid T_{i}, U=k\right) P\left(T_{i} \mid U=k\right)}{P(D \mid U=k)} \\
& =\frac{\sum_{\left(\begin{array}{c}
n \\
k-1
\end{array}\right)} P\left(D \mid T_{i, j_{1}, \ldots, j_{k-1}}, U=k\right) P\left(T_{j_{1}, \ldots, j_{k-1}} \mid T_{i}, U=k\right)}{P(D \mid U=k)} \\
& \times P\left(T_{i} \mid U=k\right) .
\end{aligned}
$$

The denominator can be written as

$$
P(D \mid U=k)=\sum_{\left(\begin{array}{l}
n \\
k
\end{array}\right)} P\left(D \mid T_{j_{1}, \ldots, j_{k}}, U=k\right) P\left(T_{j_{1}, \ldots, j_{k}} \mid U=k\right) .
$$

Assuming the initial prior is uniform, we get

$$
\begin{aligned}
& P\left(T_{i} \mid D, U=k\right) \\
& =\frac{P\left(T_{j_{1}, \ldots, j_{k-1}} \mid T_{i}, U=k\right) P\left(T_{i} \mid U=k\right)}{P\left(T_{j_{1}, \ldots, j_{k}} \mid U=k\right)} \\
& \times \frac{\sum_{\left(\begin{array}{c}
n \\
k-1
\end{array}\right)} P\left(D \mid T_{i, j_{1}, \ldots, j_{k-1}}, U=k\right)}{\sum_{\left(\begin{array}{c}
n \\
k
\end{array}\right)} P\left(D \mid T_{j_{1}, \ldots, j_{k}}, U=k\right)} \\
& =\frac{\frac{1}{\left(\begin{array}{l}
n-1 \\
k-1
\end{array}\right)} \frac{\left(\begin{array}{c}
n-1 \\
k-1
\end{array}\right)}{\left(\begin{array}{l}
n \\
k
\end{array}\right)}}{\frac{1}{\left(\begin{array}{l}
n \\
k
\end{array}\right)}} \frac{\sum_{\left(\begin{array}{c}
n \\
k-1
\end{array}\right)} P\left(D \mid T_{i, j_{1}, \ldots, j_{k-1}}, U=k\right)}{\sum_{\left(\begin{array}{c}
n \\
k
\end{array}\right)} P\left(D \mid T_{j_{1}, \ldots, j_{k}}, U=k\right)} \\
& =\frac{\sum_{\left(\begin{array}{c}
n \\
k-1
\end{array}\right)} P\left(D \mid T_{i}, T_{j_{1}}, \ldots, T_{j_{k-1}}, U=k\right)}{\sum_{\left(\begin{array}{c}
n \\
k
\end{array}\right)} P\left(D \mid T_{j_{1}}, \ldots, T_{j_{k}}, U=k\right)} .
\end{aligned}
$$

Therefore, assuming that the detections, conditioned on the location of the targets are independent of each other, we can write the above equation as

$$
\begin{aligned}
& P\left(T_{i} \mid D, U=k\right) \\
& =\quad P\left(D_{i} \mid T_{i}\right) \frac{\sum_{\left(\begin{array}{c}
n \\
k-1
\end{array}\right)} P\left(D \backslash D_{i} \mid T_{i, j_{1}, \ldots, j_{k-1}}, U=k\right)}{\sum_{\left(\begin{array}{c}
n \\
k
\end{array}\right)} P\left(D \mid T_{j_{1}, \ldots, j_{k}}, U=k\right)} .
\end{aligned}
$$

The main advantage of this formulation is that we have a very significant reduction in memory requirement as we are only storing $2 n$ numbers at each stage instead of $\left(\begin{array}{l}n \\ k\end{array}\right)$. We will later show how to exactly compute the numerator and denominator in the above equation using Newton's identities from the theory of symmetric polynomials that further leads to computational savings.

\section{B. Unknown number of Target Case}

In this case, we consider that the number of targets is unknown apriori. However, we assume that the maximum number of targets possible is $\bar{T}$. This case is considerably difficult than the previous case where the number of targets were known apriori. The belief map update proceeds in the 
following manner:

$$
\begin{aligned}
& P\left(T_{i} \mid D\right) \\
& =\sum_{j=1}^{\bar{T}} P\left(T_{i} \mid D, U=j\right) P(U=j \mid D) \\
& =\sum_{j=1}^{\bar{T}} \frac{P\left(D \mid T_{i}, U=j\right) P\left(T_{i} \mid U=j\right) P(U=j \mid D)}{P(D \mid U=j)} \\
& =\sum_{j=1}^{\bar{T}} \frac{P\left(D \mid T_{i}, U=j\right) P\left(T_{i} \mid U=j\right) P(U=j)}{P(D)} \\
& =\sum_{j=1}^{\bar{T}} \frac{P\left(D \mid T_{i}, U=j\right) P\left(T_{i} \mid U=j\right) P(U=j)}{\sum_{l=1}^{\bar{T}} P(D \mid U=l) P(U=l)} .
\end{aligned}
$$

Assuming uniform prior for $P(U=j)$, we get

$$
\begin{aligned}
& P\left(T_{i} \mid D\right) \\
& =\sum_{j=1}^{\bar{T}} \frac{P\left(D \mid T_{i}, U=j\right) P\left(T_{i} \mid U=j\right)}{\sum_{l=1}^{\bar{T}} P(D \mid U=l)} \\
& =\frac{\sum_{j=1}^{\bar{T}} P\left(D \mid T_{i}, U=j\right) P\left(T_{i} \mid U=j\right)}{\sum_{l=1}^{\bar{T}} P(D \mid U=l)} .
\end{aligned}
$$

After a few manipulations, we get

$$
\begin{aligned}
& P\left(T_{i} \mid D\right) \\
& =\frac{\sum_{j=1}^{\bar{T}} \frac{P\left(D_{i} \mid T_{i}\right)}{\left(\begin{array}{l}
n \\
j
\end{array}\right)} \sum_{\left(\begin{array}{c}
n \\
j-1
\end{array}\right)} P\left(D \backslash D_{i} \mid T_{l_{1}, \ldots, l_{j-1}}, U=j\right)}{\sum_{l=1}^{\bar{T}} \frac{1}{\left(\begin{array}{c}
n \\
l
\end{array}\right)} \sum_{\left(\begin{array}{c}
n \\
l
\end{array}\right)} P\left(D \mid T_{l_{1}, \ldots, l_{T}}, U=l\right)} .
\end{aligned}
$$

Now, for both the single and multiple target case, we need to compute

$$
P\left(D \mid T_{i_{1}, \ldots, i_{j}}, U=j\right)=\prod_{l=1}^{n} P\left(D_{l} \mid T_{i_{1}, \ldots, i_{j}}, U=j\right),
$$

where $D_{l}$ is the detection data corresponding to cell $l$. Define

$$
a_{l}=P\left(D_{l} \mid T_{i_{1}, \ldots, i_{j}}, U=j\right)=\left(\begin{array}{c}
d_{l}+m_{l} \\
d_{l}
\end{array}\right) p_{d}^{d_{l}}\left(1-p_{d}\right)^{m_{l}},
$$

if $l \in\left\{i, i_{1}, \ldots, i_{j}\right\}$ and

$$
b_{l}=P\left(D_{l} \mid T_{i_{1}, \ldots, i_{j}}, U=j\right)=\left(\begin{array}{c}
d_{l}+m_{l} \\
m_{l}
\end{array}\right) p_{f}^{d_{l}}\left(1-p_{f}\right)^{m_{l}} .
$$

otherwise. Therefore, we need an algorithm to efficiently compute terms of the form

$$
\begin{aligned}
& \sum_{\left(\begin{array}{c}
n \\
j
\end{array}\right)} P\left(D \mid T_{i_{1}, \ldots, i_{j}}, U=j\right) \\
& =\sum_{\left(\begin{array}{c}
n \\
j
\end{array}\right)=1}^{n} P\left(D_{l} \mid T_{i_{1}, \ldots, i_{j}}, U=j\right) \\
& =\sum_{\left(\begin{array}{c}
n \\
j
\end{array}\right)} a_{i_{1}}, \ldots, a_{i_{j}} b_{i_{j+1}} \ldots b_{n} .
\end{aligned}
$$

This can be written as

$$
\sum_{\left(\begin{array}{c}
n \\
j
\end{array}\right)} P\left(D \mid T_{i_{1}, \ldots, i_{j}}, U=j\right)=P_{B} \sum_{1 \leq i_{1}<\ldots<i_{j} \leq n} c_{i_{1}}, \ldots, c_{i_{j}}
$$

where $P_{B}=b_{1} \ldots b_{n}$ and $c_{i}=\frac{a_{i}}{b_{i}}$.

As one can see, computing $P\left(T_{i} \mid D\right)$ involves a larger number of operations and can considerably slow down the belief map update. We are faced with the following problem. Given $n$ numbers $c_{1}, \ldots c_{n}$ and an integer $k$, compute the symmetric polynomials

$$
J_{k}=\sum_{1 \leq i_{1}<\ldots<i_{k} \leq n} c_{i_{1}} \ldots c_{i_{k}}
$$

in an efficient manner? In the next section, we briefly review the theory of symmetric polynomials and show how the symmetric polynomials can be expressed in terms of the power sums using Newton's identities. This novel application of Newton's identities considerably reduces the number of computations needed to update the belief map.

Remark: In our setting, we are assuming that measurements are independent conditioned on target locations. In other words, measurements are not necessarily independent random variables if the location of some targets are unknown. If the measurements were independent, this would reduce to the case when there is no temporal element to the problem and we might as well consider all measurements as being made simultaneously. The problem now becomes an $O(n)$ problem with each cell being treated independently. The probability of finding a target in cell $j$ is simply computed by conditioning on the measurements made in cell $j$ only. If $d_{i_{1}}, \ldots, d_{i_{k}}$ are the measurements made in cell $j$ up to time $k$, we have

$$
P\left(T_{j} \mid d_{i_{1}}, \ldots, d_{i_{k}}\right)=\frac{P\left(d_{i_{1}}, \ldots, d_{i_{k}} \mid T_{j}\right) P\left(T_{j}\right)}{P\left(d_{i_{1}}, \ldots, d_{i_{k}}\right)}
$$

with $P\left(d_{i_{1}}, \ldots, d_{i_{k}} \mid T_{j}\right)$ being proportional to $\Pi_{i} P\left(d_{i} \mid T_{j}\right)$.

\section{Symmetric Polynomials and Newton's Identities}

A symmetric polynomial on $n$ variables $x_{1}, \ldots, x_{n}$ is a function that is invariant to any permutation of its variables. That is, the symmetric polynomials satisfy

$$
f\left(y_{1}, y_{2}, \ldots, y_{n}\right)=f\left(x_{1}, x_{2}, \ldots, x_{n}\right),
$$

where $y_{i}=x_{\pi i}$ and $\pi$ being an arbitrary permutation of the indices $1,2, \ldots, n$. The elementary symmetric polynomials 
$J_{k}\left(x_{1}, x_{2}, \ldots, x_{n}\right)$ are given by

$$
\begin{aligned}
J_{1}\left(x_{1}, x_{2}, \ldots, x_{n}\right) & =\sum_{1 \leq i \leq n} x_{i} \\
J_{2}\left(x_{1}, x_{2}, \ldots, x_{n}\right) & =\sum_{1 \leq i<j \leq n} x_{i} x_{j} \\
J_{3}\left(x_{1}, x_{2}, \ldots, x_{n}\right) & =\sum_{1 \leq i<j<k \leq n} x_{i} x_{j} x_{k}, \\
& \vdots \\
J_{n}\left(x_{1}, x_{2}, \ldots, x_{n}\right) & =\prod_{1 \leq i \leq n} x_{i} .
\end{aligned}
$$

The power sum $S_{p}\left(x_{1}, x_{2}, \ldots, x_{n}\right)$ is defined as

$$
S_{p}\left(x_{1}, x_{2}, \ldots, x_{n}\right)=\sum_{k=1}^{n} x_{k}^{p} .
$$

The relation between the symmetric polynomials $J_{k}$ and the power sums $S_{p}$ is given by Newton's identities. The first few identities are

$$
\begin{aligned}
J_{1} & =S_{1} \\
J_{2} & =\frac{1}{2}\left(S_{1}^{2}-S_{2},\right) \\
J_{3} & =\frac{1}{6}\left(S_{1}^{3}-3 S_{1} S_{2}+2 S_{3},\right) \\
J_{4} & =\frac{1}{24}\left(S_{1}^{4}-6 S_{1}^{2} S_{2}+3 S_{2}^{2}+8 S_{1} S_{3}-6 S_{4} .\right)
\end{aligned}
$$

In general, the symmetric polynomials can be computed using the following determinant

$$
J_{k}=\left|\begin{array}{cccccc}
S_{1} & 1 & 0 & 0 & \cdots & 0 \\
\frac{1}{2} S_{2} & \frac{1}{2} S_{1} & 1 & 0 & \cdots & 0 \\
\frac{1}{3} S_{3} & \frac{1}{3} S_{2} & \frac{1}{3} S_{1} & 1 & \cdots & 0 \\
\vdots & \vdots & \vdots & \vdots & \ddots & 1 \\
\frac{1}{k} S_{k} & \frac{1}{k} S_{k-1} & \frac{1}{k} S_{k-2} & \frac{1}{k} S_{k-3} & \cdots & \frac{1}{k} S_{1}
\end{array}\right| .
$$

The main advantage is that the combinatorial complexity of the original problem is reduced to polynomial complexity. All of these identities can be computed very easily using any commercial symbolic package. The main advantage of writing it in this form is that computing $S_{i}$ is much cheaper as it involves vector processing.

Though joint detection and tracking is not the subject of this paper, we feel that symmetric polynomials can be used to reduce the computational complexity. Note that Bayes recursion in multi-target detection and tracking problems is intractable in most practical applications and often is approximated.

\section{Simulation Results}

In this section, we provide simulation results for a grid of size $15 \times 15$. Note that the state space for the system for 5 targets is $4.6 \times 10^{9}$. We choose 5 target at locations $43,77,99,155,216$ at $t=0$. The initial prior is assumed to be uniform. The values of $p_{d}$ and $p_{f}$ are chosen to be 0.9
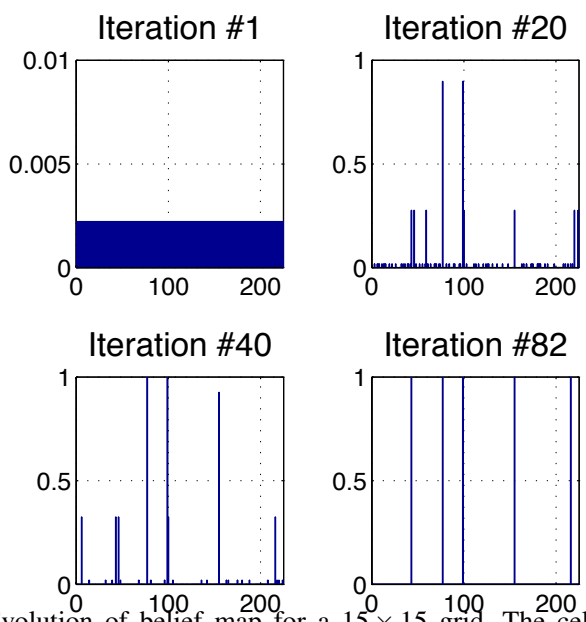

Fig. 2. Evolution of belief map for a $15^{0} \times 15$ grid. The cell number is plotted on the $x$-axis and the belief map values are plotted on the $y$-axis. After the 82nd iteration, targets are detected at location 43,77,99, 155, 216.

and 0.1 respectively. As shown in the Figure (2), the belief map converges to the expected value in about 80 iterations. The (global) teleporting scheme gives lower bounds on the detection times and serves as a baseline against which other strategies can be compared. A more realistic strategy is the local teleporting where each vehicle is moved to a neighboring cell with the maximum belief map value. Note that these strategies are optimal only when $p_{d}$ and $p_{f}$ are close to their ideal values of 1 and 0 respectively. We will be presenting quantitative results on the optimality of teleporting and other strategies in our future work. The upper and lower thresholds for the belief map entries are chosen to be $P_{u}=0.999$ and $P_{l}=0.001$ respectively.

We have also examined the effect of the number of search vehicles and $p_{d}$ on the detection times. As expected, as the number of search vehicles increases, the detection time decreases with the number of search vehicles. One such simulation result is shown in Fig. 3. However, it is interesting to note that beyond a critical value of the number of vehicles, the detection time does not change much with the number of vehicles. Such an observation can provide guidelines in choosing the number of search vehicles for a given search mission. Figure 4 shows the variation of the detection times with $p_{d}$. Once again, as expected the detection times decreases almost linearly with $p_{d}$.

We also examined the case when $p_{d}$ and $p_{f}$ are away from 1 and 0 respectively. The only thing that changes is the time to detect. The number of vehicles used was 4 . Two different cases were examined. In the first case, $p_{d}=0.9$ and $p_{f}=0.1$. The mean and variance (for 100 simulations) of the detection time were 130.7 and 5.4 respectively. For the second case, $p_{d}=0.7$ and $p_{f}=0.3$. As expected, the detection time increased. In this case, the mean and variance for the detection time were 391.1 and 33.7 respectively. 


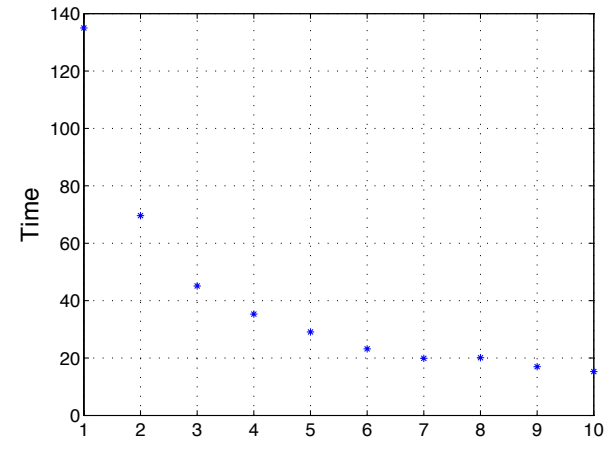

Number of vehicles

Fig. 3. Expected time versus the number of search vehicles

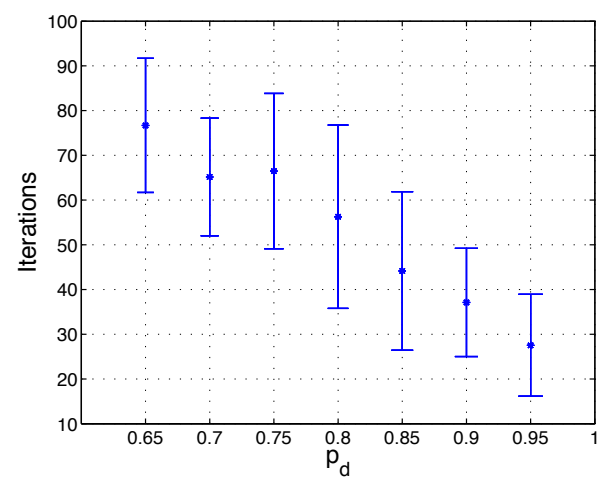

Fig. 4. Expected time (number of iterations to detect) versus $p_{d}$

\section{CONCLUSIONS}

In this paper, we have demonstrated optimal Bayesian updates for detecting unknown number of targets in a region that is divided into cells. The belief states of the system grow combinatorially and cannot be solved exactly. We develop a different formulation and show that we can indeed solve the problem exactly using results from the theory of symmetric polynomials and using the detection history. As future work, we will provide theoretical estimates of the detection times when the vehicles are globally teleported. This will provide a theoretical lower bound for expected time which can be used as a benchmark to compare other vehicle reassignment strategies based on entropy of both the belief map for target location and belief map for estimated number of targets.

\section{ACKNOWLEDGMENTS}

The authors gratefully acknowledge DARPA for funding this research. This work was supported in part by DARPA DSO under AFOSR contract FA9550-07-C-0024. The authors also thank Ozgur Erdinc for bringing [23] to their attention.

\section{REFERENCES}

[1] S. Blackman, Multiple-Target Tracking with Radar Applications. Norwood, MA: Artech House, 1986.
[2] Y. Bar-Shalom and W. D. Blair, Multitarget-Multisensor Tracking: Applications and Advances. Norwood, MA: Artech House, 2000.

[3] L. D. Stone, C. A. Barlow, and T. Corwin, Bayesian Multiple Target Tracking. Norwood, MA: Artech House, 1999.

[4] Y. Bar-Shalom and X. R. Li, Estimation and Tracking: Principles, Techniques and Software. Norwood, MA: Artech House, 1993.

[5] E.-M. Wong, F. Bourgault, and T. Furukawa, "Multi-vehicle bayesian search for multiple lost targets," in Proceedings of the IEEE international conference on robotics and automation, 2005, pp. 3180-3185.

[6] J. M. Dobbie, "A survey of search theory," Operations Research, vol. 16 , no. 3, pp. 525-537, 1968.

[7] L. D. Stone, "Search theory: a mathematical theory for finding lost objects," Mathematics Magazine, vol. 50, no. 5, pp. 248-256, 1977.

[8] _ "What's happened in search theory since the 1975 lancaster prize?" Operations Research, vol. 37, no. 3, pp. 501-506, 1989.

[9] — The theory of optimal search. Arlington, Virginia: ORSA Books, 1989.

[10] F. Bourgault, T. Furukawa, and H. F. Durrant-Whyte, "Coordinated decentralized search for a lost target in a bayesian world," in Proceedings of the IEEE/RSJ international conference on intelligent robots and systems, 2003, pp. 48-53.

[11] _ "Process model, constraints and the coordinated search strategy," in Proceedings of the IEEE international conference on intelligent robots automation, vol. 5, 2004, pp. 5256-5261.

[12] T. Furukawa, F. Bourgault, B. Lavis, and H. F. Durrant-Whyte, "Recursive bayesian search and tracking using coordinated UAVs for lost targets," in Proceedings of the IEEE/RSJ international conference on intelligent robots and systems, Orlando, Florida, 2006, pp. 25212526.

[13] M. Jun, A. I. Chaudhry, and R. D. Andrea, "The navigation of autonomous vehicles in uncertain dynamic environments : a case study," in Proceedings of the IEEE Controls and Decision Conference, vol. 4, 2002, pp. 3770-3775.

[14] M. Jun and R. D. Andrea, "Probability map building of uncertain dynamic environments with indistinguishable obstacles," in Proceedings of the IEEE American Controls Conference, vol. 4, 2003, pp. 34173422.

[15] S. M. Pollock, "A simple model of search with a moving target," Operations Research, vol. 18, no. 5, pp. 883-903, 1970.

[16] L. F. Bertuccelli and J. P. How, "Bayesian forecasting in multivehicle search operations," in AIAA Guidance, Navigation and Control Conference, Keystone, Colorado, 2004.

[17] R. Mahler, "Multi-target bayes filtering via first-order multi-target moments," IEEE Transactions on Aerospace and Electronic Systems, vol. 39, no. 4, pp. 1152-1178, 2003.

[18] G. V. Keuk, "MHT extraction and track maintenance of a target formation," IEEE Transactions on Aerospace and Electronic Systems, vol. 38, no. 1, pp. 288-295, 2002.

[19] R. Mahler, "Phd filters of higher order in target detection," IEEE Transactions on Aerospace and Electronic Systems, vol. 43, no. 4, pp. 1523-1543, 2007.

[20] B. N. Vo, S. Singh, and A. Doucet, "Sequential monte carlo methods for multi-target filtering with random finite sets," IEEE Transactions on Aerospace and Electronic Systems, vol. 41, no. 4, pp. 1224-1245, 2005.

[21] B. N. Vo and W. K. Ma, "The gaussian mixture probability hypothesis density filter," IEEE Transactions on Signal Processing, vol. 54, no. 11, pp. 4091-4104, 2006.

[22] I. G. Macdonald, Symmetric Functions and Hall Polynomials. Oxford Clarendon Press, 1995.

[23] B. Vo, B. Vo, and A. Cantoni, "Analytic implementations of the cardinalized probability hypothesis density filter," IEEE Transactions on Signal Processing, vol. 55, no. 7, pp. 3553-3567, 2007. 